\title{
AN EFFICIENT RETRIEVAL STRATEGY FOR WAVELET-BASED QUANTIZED IMAGES
}

\author{
A. Chaker ${ }^{1}$, M. Kaaniche $^{2}$, and A. Benazza-Benyahia ${ }^{1}$ \\ ${ }^{1}$ COSIM Lab., SUP'COM, Carthage Univ., \\ Cité Technologique des Communications, \\ 2080, Tunisia \\ amani.chaker@gmail.com \\ benazza.amel@supcom.rnu.tn \\ ${ }^{2}$ Université Paris-Est, \\ LIGM and UMR-CNRS 8049, \\ Champs-sur-Marne, 77454 \\ Marne-la-Vallée, France \\ mounir.kaaniche@univ-mlv.fr
}

\begin{abstract}
Recent research efforts have been devoted to the improvement of image retrieval systems when datasets are represented in a compressed form. In this context, new studies have shown that compression has a negative impact on the performances of the traditional retrieval systems. In this work, we are mainly interested in designing an efficient retrieval approach well adapted to wavelet-based compressed images. More precisely, we first propose to apply a compression scheme based on the Moment Preserving Quantization (MPQ). Then, the feature vectors will be defined in an appropriate way by focusing on the quantized subbands where some given statistical moments have been preserved. Experimental results indicate that the proposed approach outperforms the most recent one which involves the conventional uniform quantizer and constrains the query and the model images to have similar qualities during the retrieval step.
\end{abstract}

Index Terms - Content based image retrieval, wavelet domain, moment preserving quantization, feature extraction, retrieval performance.

\section{INTRODUCTION}

The recent advances in acquisition and display technologies have led to the constitution of huge image databases (DB) and digital archives. In order to reduce the storage requirements, different compression schemes have been developed such as those adopted in JPEG and JPEG2000 standards based respectively on Discrete Cosine Transform (DCT) and Wavelet Transform (WT) [1]. For instance, much attention was paid to wavelet-based compression techniques due to the sparsity of the multiscale representation and the good space frequency localization. Furthermore, the rapid increase of digital archives has allowed the widespread use of multimedia indexing systems. Indeed, Content-Based Image Retrieval (CBIR) systems aim to provide an efficient tool for managing large image databases based on their visual contents [2]. Therefore, with the increased need for both image compression and retrieval, today's CBIR systems tend to involve images not only in their original versions, but also in their compressed forms. Consequently, efficient CBIR systems should enable a fast and accurate image indexing from the stored wavelet coefficients. In this respect, several waveletbased image retrieval methods have been developed $[3,4,5]$. Their related indexing step aims at computing the features from both the original wavelet coefficients of the query and DB images (called hereafter model images). While such methods are well adapted for images which are losslessly encoded, a particular attention should be paid to the case of lossy data compression. In this case, the intuitive retrieval strategy will consist of directly computing and comparing the features extracted from the quantized wavelet coefficients of the query and model images. However, the latter techniques may not be efficient when the query and model images have different qualities (i.e. they are quantized at very different bitrates). Indeed, it has been shown that compression adversely affects the performance of color and texture image retrieval algorithms $[6,7,8]$. Therefore, a key issue is to design more efficient indexing methods that account for the quantization step. To the best of our knowledge, few works have addressed this problem $[6,9,10]$. For instance, in our recent work [10], we have improved the retrieval performances of wavelet-based compressed datasets by forcing the model and the query images to have similar qualities through a recompression strategy. More precisely, when the query and DB images are compressed at very different qualities, and before performing the feature extraction and comparison steps, we have proposed to reconstruct the high quality image and then, to quantize again the recovered WT coefficients at the bitrate of the low quality image. It is worth pointing out that a similar requantization approach has also been investigated in the context of DCT-based CBIR system [9, 11]. However, this recompression strategy has some limitations. Indeed, when the images are quantized at very low bitrates, the resulting quantization error becomes higher and therefore, the recompression technique will adversely impact the feature relevance. Furthermore, the reconstruction and the requantization steps lead to a computational overhead at the retrieval stage.

The main objective of this paper is to alleviate these shortcomings by resorting to a compression scheme that can preserve a set of statistical features of the wavelet coefficients to be quantized. To this end, we propose to apply the Moment Preserving Quantizer (MPQ) which has been designed to preserve the moments of the input signal. Furthermore, we propose to adapt the state-of-the-art moment-based feature extraction method to the preserved statistical moments of the different quantized subbands.

The rest of this paper is organized as follows. In Sec. 2, we first present the generic wavelet-based coding system as well as the most current feature extraction techniques operating in the WT domain. Then, we recall the recent image retrieval algorithm for the compressed images. In Sec. 3, we describe a novel retrieval approach by using a MPQ-based coding scheme. Finally, in Sec. 4, experimental results are given and some conclusions are drawn in Sec. 5.

\section{COMPRESSED-DOMAIN CBIR SYSTEM}

\subsection{Classical wavelet-based compression scheme}

A typical wavelet-based coding system consists of three modules namely wavelet-based transform, quantization and entropy coding. 
More precisely, a discrete wavelet decomposition is firstly applied to the original data to be encoded. To this end, a Lifting Scheme (LS)-based implementation has been introduced by Sweldens in order to construct bi-orthogonal wavelets by a simple, reversible and fast process. A generic one dimensional LS consists of applying a prediction and update steps to the input signal $a_{j}(k)$ in order to generate the detail sub-signals $d_{j+1}(k)$ and the approximation $a_{j+1}(k)$ ones:

$$
\begin{aligned}
& d_{j+1}(k)=a_{j}(2 k+1)-\mathbf{p}_{j}^{\top} \mathbf{a}_{j}(k) \\
& a_{j+1}(k)=a_{j}(2 k)+\mathbf{u}_{j}^{\top} \mathbf{d}_{j+1}(k)
\end{aligned}
$$

where $\mathbf{a}_{j}(k)$ (resp. $\mathbf{d}_{j+1}(k)$ ) is a reference vector containing some even samples (resp. detail coefficients) used in the prediction (resp. update) step, and $\mathbf{p}_{j}$ (resp. $\mathbf{u}_{j}$ ) is the prediction (resp. update) weighting vector. Such 1D structure is generally applied along the lines then the columns (or inversely) of the image in order to produce an approximation subband and three detail ones oriented horizontally, vertically and diagonally. By successively applying this procedure to the resulting approximation subband, a multiresolution representation of the input image over $J$ levels is generated. In the following, $x_{j}$ will denote the $j^{\text {th }}$ subband of size $K_{j} \times L_{j}$ with $j \in\{1, \ldots, 3 J+1\}$.

Once this multiscale transform is performed, the coefficients of each subband $x_{j}$ are quantized using an uniform quantizer with a central deadzone. Thus, for each coefficient located at position $(k, l)$, the output $\bar{x}_{j}$ of the quantizer is given by:

$$
\bar{x}_{j}(k, l)=\operatorname{sign}\left(x_{j}(k, l)\right)\left\lfloor\frac{\left|x_{j}(k, l)\right|}{q_{j}}\right\rfloor
$$

where $q_{j}$ denotes the quantization step retained in the $j^{\text {th }}$ subband. It is worth noting that a small (resp. high) $q_{j}$ value corresponds to a high (resp. low) bitrate $r_{j}$ and, results in a high (resp. low) reconstructed subband quality.

Generally, the different quantization steps $q_{1}, q_{2}, \ldots, q_{3 J+1}$ (and, hence the related bitrates $r_{1}, \ldots, r_{3 J+1}$ ) can be found by using an optimal bit allocation algorithm based on a rate-distortion criterion. Indeed, the average distortion in the WT domain is minimized subject to a constraint on the total available bitrate $R$ :

$$
R=\frac{\sum_{j=1}^{3 J+1} K_{j} L_{j} r_{j}}{\sum_{j=1}^{3 J+1} K_{j} L_{j}} .
$$

This constrained minimization problem can be solved using the Lagrangian optimization approach [12]. Finally, the quantized coefficients are entropy encoded to generate the compressed bit-stream.

\subsection{Feature extraction}

A wavelet-based CBIR consists of computing relevant features from the wavelet coefficients. The most popular and fast technique aims at retaining the first moments of the subband $x_{j}$ as a salient feature [3], especially the energy $E_{j}$ of the subband [13, 14]:

$$
E_{j}=\frac{1}{K_{j} L_{j}} \sum_{k=1}^{K_{j}} \sum_{l=1}^{L_{j}} x_{j}(k, l)^{2} .
$$

Note that several statistical features such as inertia, entropy and local homogeneity, defined on the co-occurrence matrix, can also be used $[15,14]$. Moreover, other works have been developed by resorting to a parametric modeling of the distribution of each wavelet subband [3, 14]. In particular, the generalized Gaussian distribution [4] and the Gaussian mixture model [16] were found to be well-suited for modeling the wavelet coefficients in every subband. The related feature vector of the image is built by taking the distribution parameters of all the wavelet subbands. It is worth noting that the so far proposed statistical models concern the unquantized coefficients $x_{j}$ considered as realizations of a continuous random variable. These models are no longer valid for quantized coefficients $\bar{x}_{j}$ which are samples of a discrete random variable.

Once the feature vectors of the query and model images are extracted, the retrieval step can be applied by searching in the DB the candidate model images whose feature vectors are closer to that of the query one. To this end, a similarity measure should be defined to perform this comparison. The most widely used one is the normalized Euclidean distance [14].

\subsection{Image retrieval after recompression}

Now, we assume that the original versions of the DB images and the query one are not available as they are represented in a compressed form by using the standard uniform quantizer. Thus, the related feature vectors will be computed from these lossy versions. A straightforward solution to design a CBIR consists of directly comparing the query and model images as it is generally considered in the case of unquantized data. However, recent studies $[11,10]$ have highlighted the drop of retrieval performances when these images have different qualities. For this reason, we have recently proposed to improve the CBIR system by integrating, before the feature extraction module, a pre-processing step that constrains the query and model images to have similar qualities [10]. Since the resulting quantization error at low bitrate is more important than the one obtained at high bitrate, our main idea consists of keeping the low quality image unchanged whereas the high quality one is transformed to a low quality version through a coarse requantization. More precisely, the reconstructed wavelet coefficients $\tilde{x}_{j}(k, l)$ are firstly computed from the finely quantized ones $\bar{x}_{j}(k, l)$ as follows:

$$
\tilde{x}_{j}(k, l)= \begin{cases}\left(\bar{x}_{j}(k, l)+\gamma\right) q_{j} & \text { if } \bar{x}_{j}(k, l)>0 \\ \left(\bar{x}_{j}(k, l)-\gamma\right) q_{j} & \text { if } \bar{x}_{j}(k, l)<0 \\ 0 & \text { otherwise, }\end{cases}
$$

where $0 \leq \gamma<1$ is a reconstruction parameter chosen by the decoder. Note that choosing $\gamma=0.5$ corresponds to a mid-point reconstruction as used in many encoding strategies [17]. Then, the reconstructed wavelet coefficients $\tilde{x}_{j}(k, l)$ are quantized again at the same bitrate of the image having a lower quality. Finally, the features are extracted from the requantized wavelet coefficients.

\section{PROPOSED IMAGE RETRIEVAL METHOD UNDER MPQ-BASED COMPRESSION SCHEME}

\subsection{Motivation}

Although some benefits can be drawn from the previous solution, this requantization strategy presents also some shortcomings. Firstly, when the images are coarsely quantized, the related quantization error increases and hence, the recompression will negatively affect the feature relevance. Moreover, such technique leads to a computational overhead at the retrieval stage since the reconstruction and requantization operations should be performed for each model image if the query image has the lowest quality. In order to overcome the 
drawbacks of this recompression approach, it would be interesting to preserve the image features through the compression procedure. Indeed, using a coding method where features are still preserved, even at low bitrates, can improve the retrieval system performance and increase its robustness against compression effects. Furthermore, it is well known that popular indexing methods in the WT domain consist in retaining the first moments of the wavelet subbands as salient features $[3,13]$. In such indexing framework, the solution is to guarantee that these moments (the features) are preserved by compression. This is the reason why we propose to replace the uniform quantizer (currently used in most of the wavelet-based compression methods) by an efficient one, referred to as moment preserving quantizer [18], which keeps the moments of the output equal to those of the input. It is important to note here that the MPQ has also been successfully used for image coding schemes [19].

\subsection{MPQ-based compression scheme}

Before describing the MPQ, let us first assume that the wavelet coefficients $x_{j}$ to be quantized are viewed as realizations of a random variable $X_{j}$ with a distribution function $F_{j}(\cdot)$. A $N_{j}$-level MPQ is completely defined by its $N_{j}$ output levels $\left\{y_{j, 1}, y_{j, 2}, \ldots, y_{j, N_{j}}\right\}$ and $\left(N_{j}-1\right)$ thresholds $\left\{s_{j, 1}, s_{j, 2}, \ldots, s_{j, N_{j}-1}\right\}$, with $y_{j, 1} \leq$ $s_{j, 1} \leq y_{j, 2} \leq \ldots \leq s_{j, N_{j}-1} \leq y_{j, N_{j}}$, and such that the first $\left(2 N_{j}-1\right)$ moments are preserved. Therefore, the design of the MPQ for the $j^{\text {th }}$ subband, with $j \in\{1, \ldots, 3 J+1\}$, reduces to solve the following system of equations:

$$
m_{j}^{n} \triangleq \mathrm{E}\left[X_{j}^{n}\right]=\int_{-\infty}^{+\infty} x_{j}^{n} d F_{j}\left(x_{j}\right)=\sum_{i=1}^{N_{j}} y_{j, i}^{n} P_{j, i}
$$

for every $n=1,2, \ldots, 2 N_{j}-1$, where

- $s_{j, 0} \triangleq-\infty$,

- $s_{j, N_{j}} \triangleq+\infty$

- $P_{j, i} \triangleq F_{j}\left(s_{j, i}\right)-F_{j}\left(s_{j, i-1}\right)$.

It results that the MPQ depends on the underlying distribution $F_{j}$ of the input signal. Very often, the Generalized Gaussian (GG) distribution is considered as an appropriate model of the distribution of the unquantized wavelet coefficients [4]. The GG law is defined by the probability density function $f_{j}$ given by:

$$
\forall \xi \in \mathbb{R}, \quad f_{j}(\xi)=\frac{\beta_{j}}{2 \alpha_{j} \Gamma\left(1 / \beta_{j}\right)} e^{-\left(|\xi| / \alpha_{j}\right)^{\beta_{j}}}
$$

where $\Gamma(\cdot)$ is the Gamma function, $\alpha_{j}$ and $\beta_{j}$ are respectively the scale and the shape parameters that can be easily estimated according to the classical maximum likelihood criterion [4]. Under the GG model, it can be checked that the $n^{\text {th }}$-order moments are expressed as follows:

$$
m_{j}^{n}= \begin{cases}\alpha_{j}^{n} \frac{\Gamma\left(\frac{n+1}{\beta_{j}}\right)}{\Gamma\left(1 / \beta_{j}\right)} & \text { if } n \text { is even } \\ 0 & \text { otherwise. }\end{cases}
$$

Thus, Equation (7) is a form of the Gauss-Jacobi mechanical quadrature [20]. The output levels $y_{j, i}$ of the $N_{j}$-level MP quantizer are the zeros of the $N_{j}$-th degree orthogonal polynomial associated with $F_{j}$. Note that the polynomials can be generated using the standard recursion relation for any three consecutive orthogonal polynomials [21]. The $P_{j, i}$ are Christoffel numbers and the $s_{j, i}$ and $y_{j, i}$ alternate by the separation theorem of Chebyshev-Markov-Stieltjes [20]. For more details about orthogonal polynomials, Christoffel numbers and the separation theorem, the reader is referred to [18].

Once the principle of the design of MPQ is described, a key issue is the adjustment of the value $N_{j}$ associated to the MPQ of the $j$-th subband. To this end, a bit allocation among the subbands is carried out in order to compute the levels $\left(N_{1}, N_{2}, \ldots, N_{3 J+1}\right)$ according to a rate-distortion criterion. More precisely, the average distortion in the WT domain is minimized subject to a constraint on the total available bitrate. The Lagrangian optimization approach [12] can also been used to solve this constrained minimization problem.

\subsection{Appropriate feature extraction procedure}

Unlike the energy-based feature extraction method where the second order moments of all the subbands are retained to define the descriptor, the feature vector in the context of MPQ-based coding scheme should be appropriately defined according to the $N_{j}$-level associated to the MP quantizer of each subband. More precisely, it is necessary that $N_{j} \geq 2$ in order to ensure that the second order moment of the $j^{\text {th }}$ subband is preserved. Therefore, before taking the second order moment of all the wavelet subbands as a feature vector, we have to check if the value $N_{j}$ of each subband exceeds 1. Generally, it is well known that the coarse-scale (resp. fine-scale) subbands represent large (resp. small) percentage of the total bitrate. For instance, after performing the optimal bit allocation algorithm among the different subbands, it can be noticed that the cases where the second order moment is not preserved (i.e. $N_{j}=1$ ) occur especially at low bitrates in the higher frequency subbands, whereas at middle and high bitrates, $N_{j}$ is often greater than or equal to 2 . As a result, at low bitrate (resp. middle and high bitrates), we propose to define the feature vector by taking the second order moment of only the low frequency subbands (resp. of all the subbands). Moreover, it is worth pointing out that the feature vectors of the query and model images may have different sizes when we encounter one image compressed at low bitrate and the other one one compressed at high bitrate. Therefore, during the indexing step, the descriptor vector dimension of images compressed at high bitrate will be adjusted to the size of that obtained for images compressed at low bitrate by omitting the second order moments of the high frequency subbands.

\section{EXPERIMENTAL RESULTS}

Simulations are performed on the Vision Texture (VisTex) database [22] which contains images of different natural scenes. Since we define similar textures as sub-images from a single original one, we selected 40 texture images of size $512 \times 512$ whose visual properties do not change too much over the image. Each image is then divided into 16 non-overlapping images resulting in a DB of 640 images of size $128 \times 128$. Each sub-image in the DB is used once as a query. We assume that the relevant images for each query are defined as the 16 sub-images obtained from a single original image. Moreover, in order to study the compression effect on the retrieval performance, all the images are compressed at different levels that range from low to high bitrate. The retrieval performances are evaluated in terms of precision $P R=\frac{R^{r}}{R}$ and recall $R C=\frac{R^{r}}{R^{t}}$, where $R^{r}$ is the number of output images considered as relevant, $R^{t}$ is the total number of relevant images in the database and $R$ denotes the number of returned images. The retained similarity measure is the normalized Euclidean distance.

First, Fig. 1 provides the retrieval results for the compressed database when the query and model images are respectively quantized at different bitrates $R_{Q}$ and $R_{M}$. The values of the energy of all the wavelet subbands (i.e. their second order moments $m_{j}^{2}$, 
$j \in\{1, \ldots, 3 J+1\})$ constitute the feature vector of each image. Thus, it can be noticed that recompressing the images at similar qualities (designated in the figure by AR) leads to a significant improvement in the retrieval performance compared to the case where the images are considered under different compression qualities (denoted here by UC). For instance, it is clear that the gain resulting from the recompression technique becomes much more important when the difference of qualities increases (i.e. when $\left|R_{Q}-R_{M}\right|$ is high).

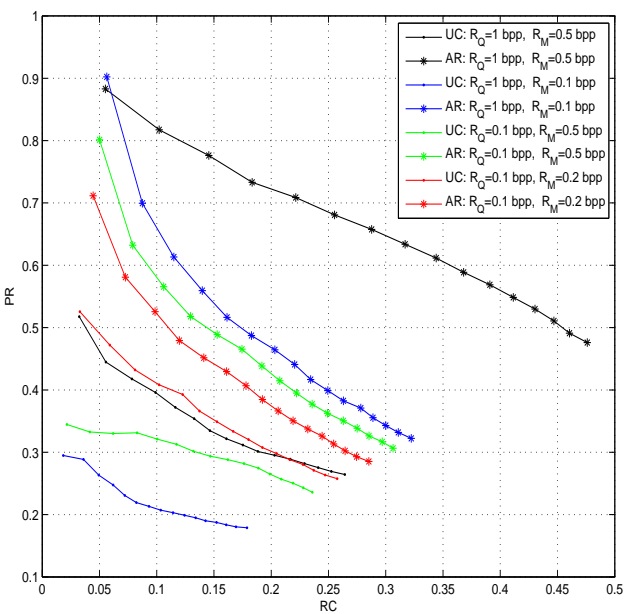

Fig. 1. Precision versus recall of wavelet-based compressed images: under compression (UC) and after recompression (AR).

Now, before comparing this recompression technique to the proposed image retrieval strategy under the MPQ-based coding method, let us study the impact of the feature extraction procedure. More precisely, we illustrate in Fig. 2 the retrieval performances when the feature vectors are defined by considering the two following cases: (i): the second order moments of all the quantized wavelet subbands are retained, (ii): the second order moments of only the quantized wavelet subbands where $N_{j} \geq 2$ are selected. Thus, it can be shown that adapting the feature extraction method as explained in Section 3.3 (i.e. case (ii)) outperforms the first feature extraction method (given by case (i)).

Finally, Fig. 3 depicts the retrieval results when query image is compressed at $R_{Q}=1 \mathrm{bpp}$ and model images are compressed at different bitrates $R_{M}$ that range from 0.1 to $1 \mathrm{bpp}$. At a similar qualities (i.e. $R_{M}=R_{Q}=1 \mathrm{bpp}$ ), it can be observed that the MPQ outperforms the standard uniform quantizer followed by the recompression method in terms of retrieval performance. Moreover, when the query and model images have different qualities, our proposed method achieves significant improvement compared to the recently reported retrieval method based on the recompression technique.

\section{CONCLUSION}

In this paper, we have presented a new approach to improve the retrieval performance of wavelet-based compressed images. For instance, we have shown that resorting to a moment preserving quantizer with an adapted feature extraction method is more interesting than using an uniform quantizer followed by the inverse quantization and recompression steps, when the database images are compressed

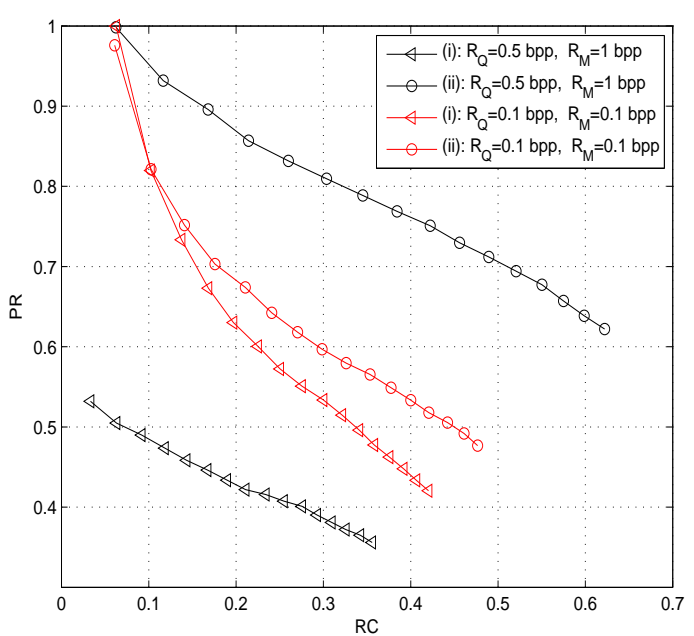

Fig. 2. Retrieval performance in terms of precision and recall when the feature vectors are defined by retaining the second order moments of (i): all the wavelet subbands, (ii): only the wavelet subbands where $N_{j} \geq 2$.

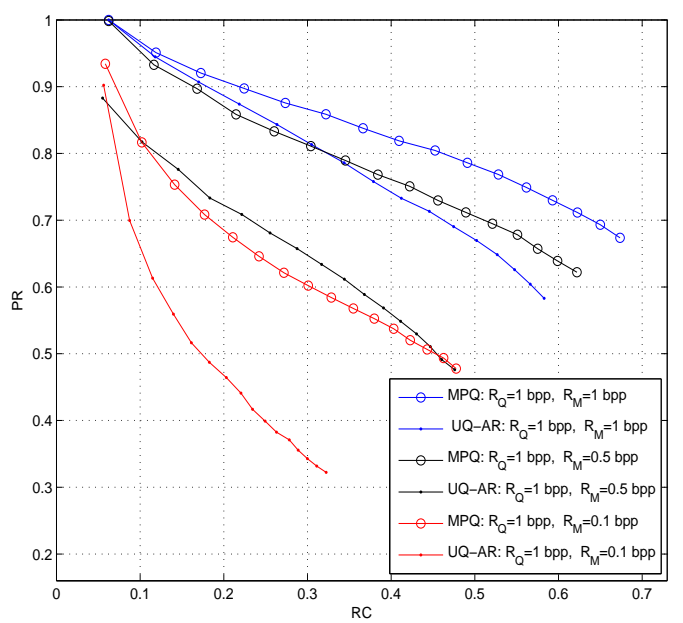

Fig. 3. Retrieval performance in terms of precision and recall when using the MPQ-based coding scheme and the Uniform Quantizer followed by the recompression method (denoted here by UQ-AR).

and used for retrieval purpose. Experimental results, carried out on the Vision Texture database, have indicated the benefits which can be drawn from the proposed method. Future works aim at designing other sophisticated features that can be more robust against the compression effects.

\section{REFERENCES}

[1] D. Taubman and M. Marcellin, JPEG2000: Image Compression Fundamentals, Standards and Practice, Kluwer Academic Publishers, Norwell, MA, USA, 2001.

[2] A. W. M. Smeulders, M. Worring, S. Santini, A. Gupta, and 
R. Jain, "Content-based image retrieval at the end of the early years," Journal of Visual Communication and Image Representation, vol. 12, pp. 1349-1380, 2000.

[3] M. K. Mandal, T. Aboulnasr, and S. Panchanathan, "Image indexing using moments and wavelets," IEEE Consumer Electr., vol. 42, no. 3, pp. 557-565, August 1996.

[4] M. N. Do and M. Vetterli, "Wavelet-based texture retrieval using generalized Gaussian density and Kullback-Leibler distance," IEEE Transactions on Image Processing, vol. 11, no. 2, pp. 146-158, February 2002.

[5] S. Sakji-Nsibi and A. Benazza-Benyahia, "Copula-based statistical models for multicomponent image retrieval in the wavelet tranform domain," in IEEE International Conference on Image Processing, Cairo, Egypt, November 2009, pp. 253 256.

[6] E. Guldogan, O. Guldogan, S. Kiranyaz, K. Caglar, and M. Gabbouj, "Compression effects on color and texture based multimedia indexing and retrieval," in IEEE International Conference on Image Processing, Barcelona, Spain, September 2003, pp. 9-12.

[7] E. Guldogan and M. Gabbouj, "DCT-based downsampling effects on color and texture-based image retrieval," in IEE European Workshop on the Integration of Knowledge, Semantics and Digital Media Technology, London, UK, November 2004.

[8] G. Schaefer, "Does compression affect image retrieval performance?," International Journal of Imaging Systems and Technology, vol. 18, no. 2-3, pp. 101-112, 2008.

[9] D. Edmundson and G. Schaefer, "Recompressing images to improve image retrieval performance," in IEEE International Conference on Acoustics, Speech and Signal Processing, Kyoto, Japan, March 2012, p. 4 pages.

[10] A. Chaker, M. Kaaniche, and A. Benazza-Benyahia, "An improved image retrieval algorithm for JPEG 2000 compressed images," in IEEE International Symposium on Signal Processing and Information Technology, December 2012.

[11] D. Edmundson, G. Schaefer, and M. E. Celebi, "Robust texture retrieval of compressed images," in IEEE International Conference on Image Processing, Orlando, USA, October 2012, pp. 2421-2424.

[12] Y. Shoham and A. Gersho, "Efficient bit allocation for an arbitrary set of quantizers," IEEE Transactions on Acoustics, Speech and Signal Processing, vol. 36, no. 9, pp. 1445-1453, September 1988.

[13] J. R. Smith and S. F. Chang, "Transform features for texture classification and discrimination in large image databases," in IEEE International Conference on Image Processing, Austin, TX, USA, November 1994, vol. 3, pp. 407- 411.

[14] G. V. Wouwer, P. Scheunders, and D. V. Dyck, "Statistical texture characterization from discrete wavelet representations," IEEE ransactions on Image Processing, vol. 8, no. 4, pp. 592598, April 1999.

[15] R. M. Haralick, K. Shanmugam, and I. H. Dinstein, "Textural features for image classification," Systems, Man and Cybernetics, IEEE Transactions on, vol. 3, no. 6, pp. 610-621, 1973.

[16] H. Yuan, X. P. Zhang, and L. Guan, "Content-based image retrieval using a gaussian mixture model in the wavelet domain," in SPIE, Visual Communications and Image Processing, Lugano, Switzerland, July 2003.
[17] M. Rabbani and R. Joshi, "An overview of the JPEG 2000 still image compression standard," Signal Processing: Image Communication, vol. 17, no. 1, pp. 3-48, 2002.

[18] E. J. Delp and O. R. Mitchell, "Moment preserving quantization," IEEE Transactions on Communications, vol. 39, no. 11, pp. 1549-1558, November 1991.

[19] E. J. Delp and O. R. Mitchell, "Image compression using block truncation coding," IEEE Transactions on Communications, vol. 27, no. 9, pp. 1335-1342, 1979.

[20] G. Szegö, Orthogonal polynomials, vol. 23, Amer Mathematical Society, 1939.

[21] D. Jackson, Fourier series and orthogonal polynomials, Dover Publications, 2004.

[22] "MIT vision and modeling group. vision texture," http: // vismod.www.media.mit.edu, [Online]. 